The authors used comparative incidence rates from the national Surveillance, Epidemiology and End Results database to determine the expected number of cases of bladder cancer in the EBRT population.

It emerged that there was no statistically significant increase in the risk of subsequent bladder cancer development for patients who had undergone EBRT. Subset analysis of patients who received adjuvant radiation did demonstrate an increased relative risk $(95 \% \mathrm{Cl})$, although this was also not statistically significant.

Based on the assumption that there was unbiased follow-up and complete ascertainment of cases, the authors conclude that the results of their study indicate that the risk of bladder cancer is not increased after EBRT for prostate cancer, and that the natural history of bladder cancer is not affected by previous prostate cancer radiation therapy.

Christine Kyme

Original article Chrouser K et al. (2005) Bladder cancer risk following primary and adjuvant external beam radiation for prostate cancer. J Urol 174: 107-111

\section{A reduced chemotherapy regimen for bladder cancer}

Lehmann et al. in Germany have published the results of a randomized, multicenter, phase III trial comparing adjuvant cisplatin plus methotrexate $(\mathrm{CM})$ with methotrexate, vinblastine, epirubicin and cisplatin (MVEC) in the adjuvant treatment of patients with resected, locally advanced bladder cancer.

Half of all patients with invasive bladder cancer die if treated with radical cystectomy alone, outlining the importance of effective chemotherapy. The authors report that the goldstandard treatment is MVEC, but the severe toxicity of this regimen has prompted them to investigate less toxic alternatives.

In this study, 327 patients with stage pT3a-4a and/or pathologic node-positive transitionalcell carcinoma of the bladder were randomized to adjuvant chemotherapy with either $\mathrm{CM}$ $(n=163)$ or MVEC $(n=164)$. Median follow-up was 42 months. The study was designed as a noninferiority trial, and the primary endpoint was progression-free survival. The equivalence margin was set at 0.15 , with the upper bound of the hazard ratio at 1.5077 .
Five-year progression-free survival rates were $46.3 \%$ for the CM arm and $48.8 \%$ for the MVEC arm. Leucopenia, the most common toxic effect, was significantly higher in the MVEC arm.

The authors conclude that the $\mathrm{CM}$ regimen is less toxic than MVEC and cannot be considered substantially inferior in terms of efficacy. However, they also note that these results must be assessed in light of the equivalence margin of 0.15 .

Tamsin Osborne

Original article Lehmann J et al. (2005) Adjuvant cisplatin plus methotrexate versus methotrexate, vinblastine, epirubicin, and cisplatin in locally advanced bladder cancer: results of a randomized, multicenter, phase III trial (AUO-AB 05/95). J Clin Oncol 23: 4963-4974

\section{Hormonal function and fertility in survivors of testicular cancer}

A UK study has shown that survivors of testicular cancer have an increased frequency of hormonal dysfunction, but that most men remain fertile at long-term follow-up.

The trial studied 608 men, 5 years after diagnosis of testicular cancer, to determine the long-term effects of treatment on fertility, gonadal and sexual function. The participants were divided into four cohorts according to treatment received: orchidectomy alone (S), orchidectomy plus chemotherapy (C), orchidectomy plus radiotherapy $(\mathrm{R})$ or orchidectomy plus chemotherapy and radiotherapy (C/RT). The men completed three questionnaires and had hormone measurements taken.

The study revealed that there was significant elevation of follicle-stimulating hormone (FSH) and luteinizing hormone, and a significant decrease in testosterone in all of the groups. In particular, testosterone was low in the C/RT group, FSH was higher in the $\mathrm{C}$ group, and luteinizing hormone was higher in both the $C$ and $\mathrm{R}$ groups. When compared with baseline measures, there was a significant increase in FSH and a decrease in testosterone after treatment for testicular cancer. Low testosterone levels were associated with general impairment in quality-of-life scores for physical and role functioning, although $83 \%$ of participants reported satisfactory sexual function. Of the $30 \%$ of men who attempted conception after treatment, $77 \%$ reported success without intervention and 5\% were successful after fertility treatment. 